\title{
Alterations in food reward regarding bariatric surgery type and weight loss outcomes: an exploratory study.
}

\author{
Erika Guyot ${ }^{1,2,3}$, Julie-Anne Nazare ${ }^{2}$, Pauline Oustric ${ }^{4}$, Maud Robert ${ }^{5}$, Emmanuel Disse ${ }^{1,2}$, Anestis Dougkas ${ }^{3,+}$ and \\ Sylvain ICETA ${ }^{1,6,+, *}$
}

\begin{abstract}
1. Department of Endocrinology Diabetes and Nutrition - Integrated Center for Obesity, Hospices Civils de Lyon, Lyon-Sud Hospital, 69310, Pierre-Bénite, France; +33 (0)4 788657 86. erikaguyot.pro@gmail.com, emmanuel.disse@chu-lyon.fr

2. Centre de Recherche en Nutrition Humaine Rhône-Alpes (CRNH-RA), Unité INSERM 1060, Laboratoire Centre Européen Nutrition et Santé (CENS), CarMeN, Université Claude Bernard Lyon 1, 165, Chemin du Grand Revoyet, 69310, Pierre-Bénite, France; +33 (0)4 262359 17. julie-anne.nazare@univ-lyon1.fr 3. Institut Paul Bocuse Research Center, 69130, Ecully, France; +33 (0)4 721802 20. anestis.dougkas@institutpaulbocuse.com

4. School of Psychology, University of Leeds, Leeds, LS2 9JT, UK; +44 (0113) 343 3738. pspjo@leeds.ac.uk 5. Department of Digestive and Bariatric Surgery, Integrated Center for Obesity, Hospices Civils de Lyon, Hôpital Edouard Herriot, 69437 Lyon, France; CarMeN Laboratory, INSERM 1060, Lyon, France; Université Claude Bernard Lyon 1, Lyon, France; +33 (0)4 721162 63. maud.robert@chu-lyon.fr 6. Centre de Recherche de l'Institut Universitaire de Cardiologie et de Pneumologie de Québec-Université Laval, 2725, Chemin Sainte-Foy, Quebec, QC G1V 4G5, Canada; +1 (418) 656-8711. sylvain.iceta.1@ulaval.ca

* These authors contributed equally to this work and shared last co-authorship

* Correspondence: sylvain.iceta.1@ulaval.ca; Tel.: +1 (418) 656-8711
\end{abstract}

\begin{abstract}
Changes in food preferences after bariatric surgery may alter its effectiveness as a treatment for obesity. We aimed to compare food reward for a comprehensive variety of food categories between patients who received a sleeve gastrectomy (SG) or a Roux-en-Y gastric bypass (RYGB) and to explore whether food reward differs according to weight loss. In this cross-sectional exploratory study, food reward was assessed using the Leeds Food Preference Questionnaire (LFPQ). We assessed liking and wanting of eleven food categories. Comparisons were done regarding type of surgery and Total Weight Loss (TWL; based on tercile distribution). Fifty-six patients (30 SG and 26 RYGB) were included (women: 70\%; age: 44.0 (11.1) y). Regarding the type of surgery, scores were not significantly different between SG and RYGB, except for 'non-dairy products - without color' explicit liking $(p=0.04)$. Regarding TWL outcomes, explicit liking, explicit wanting and implicit wanting, scores were significantly higher for Good responders than Low responders for 'No meat High fat' (post-hoc corrected p-value: 0.04, 0.03 and 0.04, respectively). Together, our results failed to identify major differences in liking and wanting regarding the type of surgery and tended to indicate that higher weight loss might be related to a higher reward for high protein-content food. Rather to focus only on palatable foods, future studies should also consider a broader range of food items, including protein reward.
\end{abstract}

Keywords: Food reward; Liking; Wanting; Food preferences; Bariatric surgery; Eating behavior; Total Weight Loss

\section{Introduction}

Bariatric surgery is considered the most effective treatment in case of severe complicated or morbid obesity, to achieve sustained weight loss, reduce comorbidities and mortality [1-3]. The most commonly performed surgical techniques are sleeve gastrectomy (SG) and Roux-en-Y gastric bypass (RYGB) [4]. Both types of procedures give similar weight loss patterns at 5-year follow-up [5]. 
Bariatric surgery has a direct effect on food intake, including reducing ingested volume [6] and total energy intake [6,7], as well as reducing hunger and increasing satiety [8]. In addition, our recently published systematic review and meta-analyses found that food preferences change after bariatric surgery in terms of macronutrients distribution, with higher contributions of proteins and lower contributions of lipids in the total energy intake postoperatively [9]. Food preferences also change in terms of food selection with a higher consumption of healthy foods and a lower consumption of highly palatable foods. Finally, it changes in terms of food appreciation with an overall lower hedonic rating postoperatively compared to before the operation [9].

The attribution of a hedonic value to foods is the result of the contribution of two domains: the sensory and the reward domains. The alterations of the sensory domain (gustation and olfaction) after bariatric surgery have been examined in recent systematic reviews $[10,11]$. Of importance, Nielsen et al. found that patients with RYGB have a higher sweet taste sensitivity [12]. Moreover, a recent publication by our research team has demonstrated that participants with and without taste or smell alterations have different food preferences [13]. On the other hand, food reward is a constantly evolving concept that includes different models. Berridge et al. described food reward as based on two distinct components [14]. The first component is 'liking' and is related to the pleasure and the sensory properties of foods. The second component is 'wanting', which is related to the motivation to eat. In turn, wanting can be divided in two components: 'explicit wanting', which can be conceptualized as the conscious motivation to eat, and 'implicit wanting' that is more related to the subliminal level (i.e., cue-elicited motivation to eat) [1518]. Interestingly, a post-surgery decrease in neuronal activity in brain areas corresponding to the reward system has been described [19-23]. Furthermore, liking and wanting scores (based on a 5-point Likert type scale on the questions "How much do you want to eat this food now?" and "How much do you like this food in general ?") were diminished after RYGB for foods high in fat using food pictures displayed during an fMRI paradigm [24].

Overall, while food reward is described as dysfunctional in the case of obesity [25,26], its alterations in relation to bariatric surgery remains to be explored. Several factors including appearance, texture, taste and the nature of foods could influence food preferences in the general population [27] and in patients after bariatric surgery [28]. However, no study explored food reward (liking and wanting) for a comprehensive range of food items postoperatively. RYGB and SG might give similar weight loss patterns at 5-year follow-up [5]. However, it has been suggested that RYGB would be more rapidly effective and lead to a higher initial weight loss and that this may be related to specific food reward changes induced by RYGB [29]. However, most of the studies so far failed to separate and explore the impact of the type of surgery on food preferences given that the analysis was conducted on only one type of surgery or by combining patients with different types of surgery. Finally, no study explored food reward in relation to weight loss status after bariatric surgery.

The aim of the present exploratory study was to compare food reward for extended various food categories between i) patients who received SG or RYGB and ii) according to weight loss status. We hypothesized that i) liking and wanting scores are not different between patients with RYGB and SG and that ii) liking and wanting scores are higher in participants with a poor weight loss response, especially regarding high carbohydrates and high fat products.

\section{Materials and Methods}

2.1. Subjects 
From June 2018 to July 2019, participants hospitalized in our bariatric surgery tertiary care center for a routine post-bariatric surgery follow-up day care $(6,12$ or 24 months after a SG or a RYGB) were eligible. For this cross-sectional study, inclusion criteria were: age between 18 to 65 years old, BMI between 18.5 and $60 \mathrm{~kg} \cdot \mathrm{m}^{-2}$. Patients with psychiatric comorbidities, under psychotropic treatments or having food evictions, as well as those unable to give their consent and understanding French were excluded. These results are part of a larger study (including non-operated participants) that was approved by a national ethic Committee (2017-A02953-50, on January 16th, 2018) and was registered in Clinical Trials.gov (NCT03486210). Due to methodological issues (inconsistent fasting time), the control group (i.e., participants with obesity without surgery) has not been considered in the present publication.

\subsection{Data collection}

\subsubsection{Food reward}

Among the various tools that exist to measure food reward, we used the Leeds Food Preference Questionnaire (LFPQ) [17], in a version adapted by Van der Meij et al. [30] as it gives a behavioral measure of food reward quantifying both explicit liking and implicit wanting. Explicit liking was measured using Visual Analog Scales (VAS) on the question "How pleasant would it be to taste some of this food now?". Implicit wanting was computed using a forced choice task during which a spontaneous choice must have been made between all combinations of food items presented consecutively in pairs. An implicit wanting score for a given food category is calculated from the frequency of choice and non-choice of this food category and the reaction time of the participants [18]. This score ranges between -100 and 100 and is interpreted in relation to the scores of the other food categories evaluated in the task. A positive score indicates that the considered food category was chosen more often and faster than the others. A negative score indicates the opposite.

Although the LFPQ as adapted by Van der Meij [30] was initially intended for use among the elderly, this version seemed relevant to explore food preferences among patients with a bariatric surgery. In particular, it was composed of 6 stages which allowed us to assess liking and wanting for 11 food categories varying in nutritional composition (high/low in carbohydrates, high/low in fat, high/low in protein, dairy/nondairy, with/without fiber, with/without meat) and/or in taste (savory/sweet), appearance (with/without color, with/without variation, with/without sauce) and texture (solid/fluid) (Table 1). These food categories were combined within a task (i.e., high fat - sweet, high fat - savory, low fat - sweet, low fat - savory).

We adapted this version for its use in a French population by translating the instructions from English to French and adapting the food items to French eating habits [18]. The new set of food pictures was validated by asking 20 health professionals specialized in nutrition or dietetics and 20 non-specialized counterparts whether the food pictures correspond to what they can eat in France using a 9-point Likert-type scale where 1 represented 'Not at all' and 9 'Extremely'. Food items with a score $\leq 5$ were considered not representative of French eating habits and were replaced. The list of food items used is available in Table 1.

\subsubsection{Other covariates}

Information on gender, age, and body mass index (BMI) $\mathrm{kg} \cdot \mathrm{m}^{-2}$ before surgery and at assessment time point were collected based on the medical record of the patients. Patients self-reported having a budget constraint to purchase food and smoking status. Ratings of hunger, fullness and desire to eat were assessed immediately prior to processing the LFPQ using visual analog scales $(100 \mathrm{~mm})$ from 'Not at all' to 'Extremely' of the following questions: "How hungry do you feel now?", "How full do you feel now?" and "How strong is your desire to eat now?". Time since the last meal was also recorded. The weight loss results were express as a percentage of the total weight loss (\%TWL $=[($ Initial Weight $)-$ (Postoperative Weight)] / [(Initial Weight) $] \times 100)$. The \%TWL metric has been considered 
a better metric than the percentage excess weight loss because its calculation does not need to define an ideal body weight and did not differ between lower and higher baseline BMI [31] and is a better predictor of metabolic outcome [32,33].

Table 1. Dichotomous food categories tested with the Leeds Food Preference questionnaire.

\begin{tabular}{ccc}
\hline Task & Studied dimensions & Combined food categories \\
\hline \multirow{4}{*}{1} & High carbohydrates (HC) / Low carbohydrates (LC) & HC-SO \\
& Solid (SO) / Fluid (FL) & HC-FL \\
& & LC-SO \\
2 & LC-FL \\
& Dairy (DA) / Nondairy (ND) & DA-CO \\
& Color (CO) / No color (NC) & DA-NC \\
& & ND-CO \\
3 & High fat (HF) / Low fat (LF) & ND-NC \\
& Savory (SA) / Sweet (SW) & HF-SA \\
& & HF-SW \\
& & LF-SA \\
4 & Fiber (FI) / No fiber (NF) & LF-SW \\
& Sauce (SC) / No sauce (NS) & FI-SC \\
& & FI-NS \\
& & NF-SC \\
5 & Meat (ME) / No meat (NM) & NF-NS \\
& High fat (HF) / Low fat (LF) & ME-HF \\
& & ME-LF \\
& & NM-HF \\
& & NM-LF \\
& High protein (HP) / Low protein (LP) & HP-VA \\
& Variation (VA) / No variation (NV) & HP-NV \\
& & LP-VA \\
& & LP-NV \\
\hline
\end{tabular}

The tasks were administered to patients in a random order.

\subsection{Statistical analysis}

General characteristics, appetite sensations and time since the last meal of patients were compared using independent t-test, Pearson's $\chi^{2}$ test or Fisher's exact test for normal continuous, categorical variables without or variables with at least one expected frequency in a fourfold table less than 5, respectively. Mann-Whitney U test was performed to compare non-normal continuous variable (i.e., time since the surgery).

In the present study, patients having a wanting score $<-100$ and $>100$ in a food category were considered as outliers and results for the food category were excluded from the analyses. Explicit liking, explicit wanting and implicit wanting scores for all the studied food categories were compared between the groups of patients (SG and RYGB) using student-t test. We also compared liking and wanting scores between patients at 6-, 12- and 24-month follow-up using one-way ANOVA. As mean and range for \%TWL were expected to differ at 6-, 12- and 24-month after bariatric surgery, we calculated the tercile separately for each post-surgery follow-up duration group (6-, 12- and 24- months). Participants in the first tercile $(<33 \%)$ were considered as Low responders, in the middle tercile $(33-66 \%)$ as Middle responders and, in the upper tercile $(>66 \%)$ as Good responders. All participants were analyzed together using one-way ANOVA. Post-hoc analyses were Bonferroni corrected using the SPSS software post-hoc option and post-hoc corrected $\mathrm{p}$-value provided for all ANOVA p-value $<0.1$. 
Data are presented as mean (SD). All the statistical analyses were conducted using SPPS software version 26.0.0.1 for Mac. All tests were two-sided and $\mathrm{p}<0.05$ was considered significant.

\section{Results}

\subsection{Characteristics of the subjects}

In the study, 61 patients were initially recruited. Five participants were excluded from the study ( 2 for antidepressant medication, 1 for actual major depressive disorder, and 2 for a follow up duration $>24$ months). A total of 56 participants (30 SG and 26 RYGB) were included in the present analysis. Table 2 presents the characteristics of the patients according to their surgery group.

Overall, 75\% of the patients were women. Patients with RYGB were significantly older $(50.5 \mathrm{y}, \mathrm{SD}=9.0)$ compared to those with SG $(38.3 \mathrm{y}, \mathrm{SD}=9.6)$. The mean BMI before surgery of the SG group $\left(45.5 \mathrm{~kg} \cdot \mathrm{m}^{-2}, \mathrm{SD}=5.9\right)$ was significantly higher compared to the RYGB group $\left(41.4 \mathrm{~kg} \cdot \mathrm{m}^{-2}, \mathrm{SD}=4.4\right)$. The number of participants for each follow-up duration was similar between SG ( $\mathrm{n}=10$ for 6-, 12- and 24- months) and RYGB ( $\mathrm{n}=10$ for 6months, $\mathrm{n}=7$ for 12 -months, and $\mathrm{n}=9$ for 24 -months; $\mathrm{p}=0.862$ ). The other parameters, including \%TWL, appetite sensations scores, were not significantly different between the two groups of surgery.

Table 2. Socioeconomic and behavioral characteristics of the 56 included patients according to their operative status.

\begin{tabular}{ccccc}
\hline & $\begin{array}{c}\text { All } \\
\mathrm{n}=56\end{array}$ & $\begin{array}{c}\text { SG } \\
\mathrm{n}=30\end{array}$ & $\begin{array}{c}\text { RYGB } \\
\mathrm{n}=26\end{array}$ & p-value \\
\hline Sociodemographic data & & & & \\
\hline Women (\%) & $75.0(\mathrm{n}=42)$ & $76.7(\mathrm{n}=23)$ & $73.1(\mathrm{n}=19)$ & 0.757 \\
Age (yr) & $44.0(11.1)$ & $38.3(9.6)$ & $50.5(9.0)$ & $<0.001$ \\
Smoking status (\%) & $19.6(\mathrm{n}=11)$ & $20.0(\mathrm{n}=6)$ & $19.2(\mathrm{n}=5)$ & 0.942 \\
Food budget constraint (\%) & 14.3 & 10.0 & 19.2 & $0.451^{\mathrm{a}}$ \\
\hline Anthropometric data & & & \\
\hline BMI Before surgery(kg.m-2) & $43.6(6.0)$ & $45.5(5.9)$ & $41.4(4.4)$ & 0.011 \\
Body weight (kg) & $87.8(17.8)$ & $88.2(20.3)$ & $87.3(14.7)$ & 0.843 \\
BMI (kg.m-2) & $31.4(4.5)$ & $31.5(4.5)$ & $31.2(4.6)$ & 0.846 \\
\%TWL & $27.7(7.3)$ & $30.6(6.3)$ & $24.3(7.0)$ & $<0.001$ \\
\hline Appetite sensations & & & & 0.196 \\
\hline Mean hunger (mm) & $21.0(25.7)$ & $25.2(28.7)$ & $16.2(21.5)$ & 0.909 \\
Mean fullness (mm) & $68.3(28.0)$ & $68.7(27.6)$ & $67.8(29.0)$ & 0.252 \\
Mean desire to eat (mm) & $22.2(24.8)$ & $25.6(28.2)$ & $18.2(19.8)$ & 0.434 \\
\hline Time since last meal (min) & $134(214)$ & $154(237)$ & $109(184)$ & \\
\hline & & & & \\
\hline
\end{tabular}

Abbreviations: SG: group with a sleeve gastrectomy; RYGB: group of patients with a Roux-en-Y gastric bypass; BMI : Body Mass Index; \%TWL: Percentage of Total Weight Loss. Data are presented as mean (SD), or percentage (number). $p$ values are for the differences between patients with SG and RYGB on the basis of student-t test and Pearson's $\chi^{2}$ test; a indicates that Fisher's exact test or Mann-Whitney U test were used.

\subsection{Relationship between liking and wanting for foods and type of bariatric surgery}

Explicit liking, explicit and implicit wanting scores regarding surgery type are illustrated in Figure 1 and detailed in supplementary Table S1. Considering all the studied food categories, scores were not significantly different between SG and RYGB, except for 'non-dairy products - without color' explicit liking (26.5, SD = 24.7 for SG and 14.8, SD = $15,7$ for RYGB; $p=0.038)$. We also observed a trend for a significant difference for 'nondairy products - without color' implicit wanting $(-17.0, \mathrm{SD}=23.7$ for SG and -27.6, SD = 
21.7 for RYGB; $\mathrm{p}=0.089)$, 'non-dairy products - no color' explicit liking $(28.3, \mathrm{SD}=23.5$ for SG and 18.5, SD = 16.7 for RYGB; $\mathrm{p}=0.087$ ), and for foods 'low in carbohydrates - fluid' implicit wanting $(-19.5, \mathrm{SD}=32.0$ for $\mathrm{SG}$ and $-2.3, \mathrm{SD}=32.8$ for $\mathrm{RYGB} ; \mathrm{p}=0.053)$. A negative implicit wanting score means that the food category items were more often rejected relatively to the items in the other food categories. Moreover, explicit liking, explicit wanting and implicit wanting scores for each follow-up duration (6,12 and 24 months) were not significantly different for any of the food categories (data not shown).

\subsection{Relationship between liking and wanting for foods regarding weight loss outcomes}

Regarding comparison between \%TWL groups, Bonferroni post-hoc test revealed that differences were significant only between Low and Good responders. Detailed results are available in Table 3, 4 and 5 (characteristics of the subjects for each tercile are presented in Table S2).

Scores were significantly higher for Good responders than Low responders for 'No meat - High fat' and this for the three types of measures, explicit liking, explicit wanting and implicit wanting (post-hoc corrected p-value: $0.037,0.033$ and 0.043 , respectively). Regarding 'High protein -Variation' items, explicit liking was higher in Good responders than in Low responders (post-hoc corrected p-value $=0.030$ ).

Regarding implicit wanting scores, more differences were found between Good and Low responders. In addition to the higher scores mentioned above for the 'No meat - High fat' category, Good responders also had significant higher scores than Low responders for 'Low carb - Solid' (post-hoc corrected p-value $=0.011$ ) and tended to be significantly higher for 'High protein - No variation' items (post-hoc corrected $p$-value $=0.061$ ). A tend to significant difference was also observed for 'Low protein - Variation' items with Good responders having lower scores than Low responders. Taking together, these results suggest that participants with higher \%TWL tended to present higher implicit wanting for food items with higher protein contents. 
Figure 1. Relationship between a) explicit liking, b) explicit wanting and c) implicit wanting for foods and bariatric surgery type.

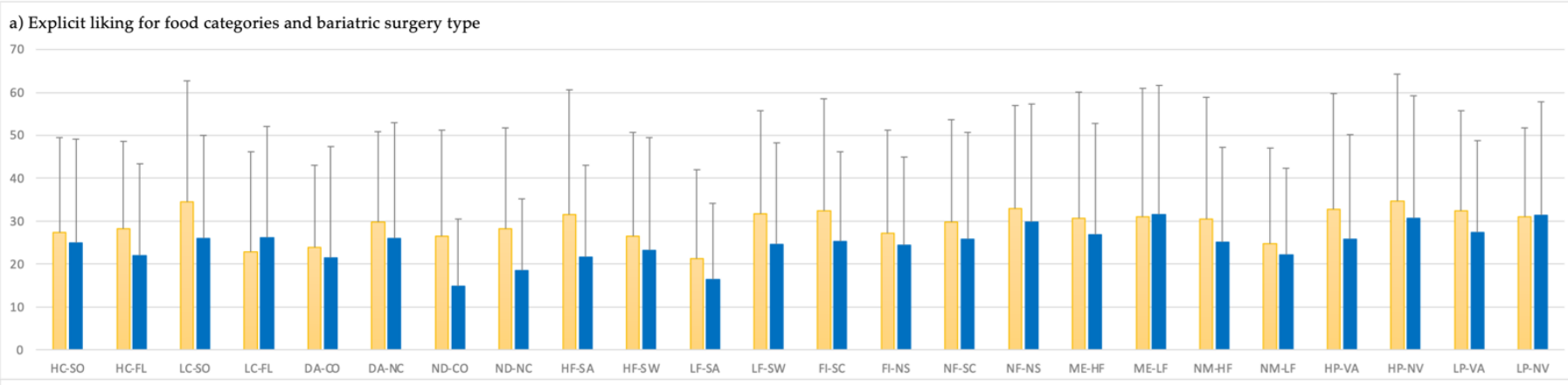

b) Explicit wanting for food categories and bariatric surgery type

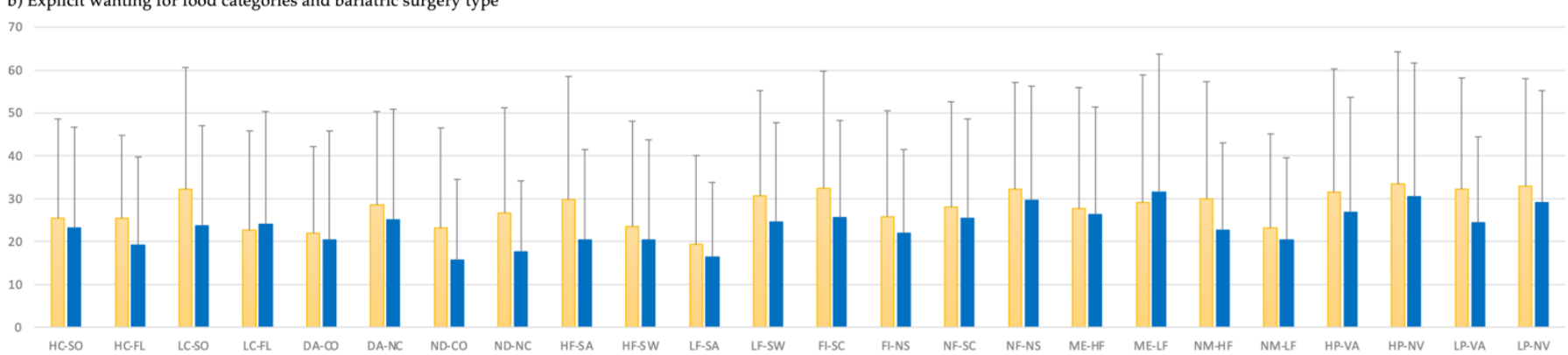

c) Implicit wanting for food categories and bariatric surgery type

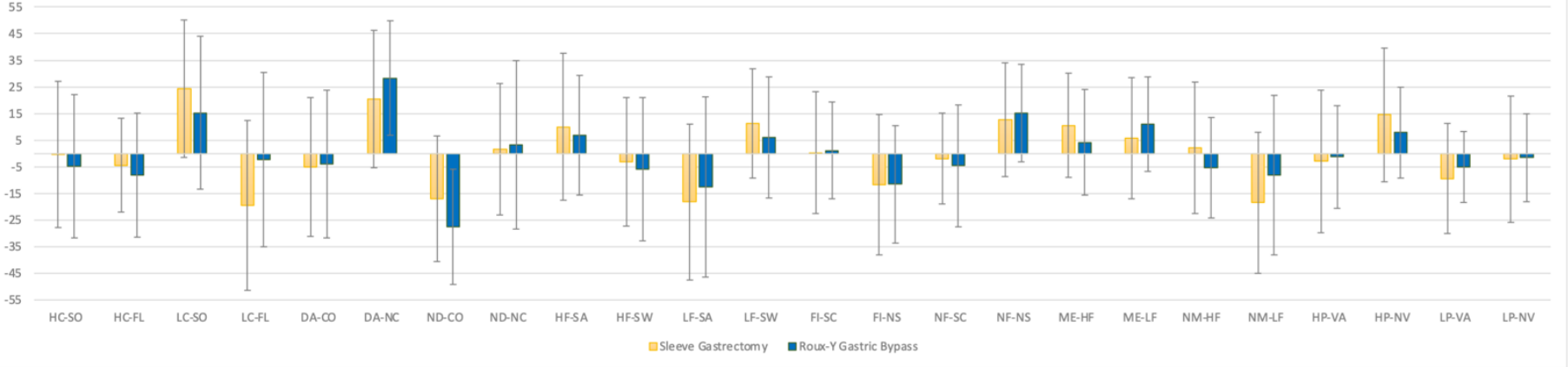

Abbreviations: CO, color; DA, dairy; FI, fiber; FL, fluid; HC, high carbohydrate; HF, high fat; HP, high protein; LC, low carbohydrate; LF, low fat; LP, low protein; ME, meat; NC, no color; ND, nondairy; NF, no fiber; NM, no meat; NS, no sauce; NV, no variation; OB: non-operative patients with obesity; SA, savory; SC, sauce; SO, solid; SW, sweet; VA, variation. Data are presented as mean (SD). 
Table 3: Relationship between explicit liking for foods regarding percentage of Total Weight Loss tercile groups.

High carb - Solid

High carb - Fluid

Low carb - Solid

Low carb - Fluid

Dairy - Color

Dairy - No color

Nondairy - Color

Nondairy - No color

High fat - Savory

High fat - Sweet

Low fat - Savory

Low fat - Sweet

Fiber-Sauce

Fiber - No sauce

No fiber - Sauce

No fiber - No sauce

Meat - High fat

Meat - Low fat

No meat - High fat

No meat - Low fat

High protein - Variation

High protein - No variation

Low protein - Variation

Low protein - No variation

\section{Low responders}

$$
\begin{aligned}
& <33 \% \\
& n=18
\end{aligned}
$$

Middle responders

$$
\begin{gathered}
33-66 \% \\
n=19
\end{gathered}
$$

mean $S D$

$22.2 \pm 20.0$

$19.2 \pm 15.7$

$21.4 \pm 23.6$

$20.3 \pm 18.2$

$17.0 \pm 14.5$

$21.1 \pm 14.8$

$16.6 \pm 19.1$

$19.3 \pm 19.0$

$19.0 \pm 23.5$

$19.5 \pm 20.1$

$9.7 \pm 10.1$

$22.8 \pm 19.7$

$21.3 \pm 18.1$

$19.2 \pm 13.6$

$21.5 \pm 19.5$

$27.6 \pm 26.3$

$18.7 \pm 22.6$

$22.5 \pm 24.1$

$18.4 \pm 18.8$

$16.9 \pm 15.5$

$18.6 \pm 22.9$

$23.9 \pm 29.1$

$24.6 \pm 20.2$

$24.3 \pm 21.3$

$29.1 \pm 24.5$

$30.4 \pm 25.5$

$30.8 \pm 31.4$

$22.3 \pm 23.9$

$28.6 \pm 27.2$

$20.4 \pm 19.2$

$20.2 \pm 18.8$

$25.2 \pm 22.0$

$26.2 \pm 24.5$

$22.4 \pm 20.6$

$29.3 \pm 27.4$

$30.4 \pm 26.4$

$29.1 \pm 26.2$

$25.9 \pm 24.1$

$30.5 \pm 27.3$

$28.6 \pm 27.2$

$31.5 \pm 30.6$

$25.7 \pm 21.3$

$25.6 \pm 23.1$

$29.2 \pm 25.8$

$31.2 \pm 27.2$

$30.0 \pm 22.7$

$32.3 \pm 25.0$

\section{Good responders}

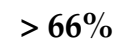

0.581

$27.2 \pm 21.2$

0.323

$39.5 \pm 28.1$

0.113

$21.9 \pm 21.3$

0.373

$28.5 \pm 25.7$

0.303

$33.7 \pm 26.0$

0.290

$26.0 \pm 26.0$

0.433

$31.4 \pm 23.8$

0.147

$36.2 \pm 30.2$

0.126

$28.8 \pm 29.8$

0.521

$24.6 \pm 22.3$

$0.040^{a}$

$32.7 \pm 24.4$

0.458

$35.3 \pm 25.0$

0.196

$29.0 \pm 24.4$

0.309

$36.1 \pm 26.9$

0.166

$36.0 \pm 23.6$

0.602

$38.7 \pm 30.2$

$0.087^{\mathrm{b}}$

$39.3 \pm 32.9$

0.232

$39.3 \pm 31.5$

$0.038^{c}$

$27.9 \pm 23.2$

0.259

$40.3 \pm 25.1$

$0.035^{\mathrm{d}}$

$42.9 \pm 28.7$

0.129

$35.2 \pm 24.0$

0.366

a: post-hoc analyses indicate a trend for a significant difference only between Low and Good responders with a Bonferroni corrected $\mathrm{p}$-value $=0.055 ; \mathrm{b}$ : post-hoc analyses indicate $\mathrm{a}$ trend for a significant difference only between Low and Good responders with a Bonferroni corrected p-value $=0.084$; c: post-hoc analyses indicate a significant difference only between Low and Goodd responders with a Bonferroni corrected $\mathrm{p}$-value $=0.037$; $\mathrm{d}$ : post-hoc analyses indicate a significant difference only between Low and Good responders with a Bonferroni corrected p-value $=0.030$. 
Table 4: Relationship between explicit wanting for foods regarding percentage of Total Weight Loss tercile groups.

\section{Low responders}

mean $S D$

High carb - Solid

High carb - Fluid

Low carb - Solid

Low carb - Fluid

Dairy - Color

Dairy - No color

Nondairy - Color

Nondairy - No color

High fat - Savory

High fat - Sweet

Low fat - Savory

Low fat - Sweet

Fiber-Sauce

Fiber - No sauce

No fiber - Sauce

No fiber - No sauce

Meat - High fat

Meat - Low fat

No meat - High fat

No meat - Low fat

High protein - Variation

High protein - No variation

Low protein - Variation

Low protein - No variation

$$
<33 \%
$$$$
n=18
$$

Middle responders

$$
\begin{gathered}
33-\mathbf{6 6} \% \\
n=19
\end{gathered}
$$

$20.6 \pm 21.5$

$17.4 \pm 15.7$

$19.9 \pm 22.8$

$18.5 \pm 18.6$

$14.8 \pm 12.8$

$21.1 \pm 16.7$

$14.4 \pm 16.8$

$17.4 \pm 16.6$

$17.0 \pm 21.8$

$18.1 \pm 21.1$

$9.2 \pm 8.5$

$23.2 \pm 21.1$

$22.8 \pm 20.0$

$16.9 \pm 13.1$

$19.5 \pm 19.0$

$25.8 \pm 23.2$

$18.4 \pm 22.3$

$22.4 \pm 26.7$

$16.6 \pm 17.1$

$14.3 \pm 13.6$

$18.1 \pm 22.3$

$21.8 \pm 27.6$

$22.9 \pm 18.5$

$24.0 \pm 19.7$

mean $S D$
Good responders

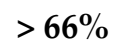

$$
\text { mean } S D
$$

$23.2 \pm 21.4$

0.532

$23.4 \pm 19.1$

0.376

$38.0 \pm 29.1$

0.103

$22.9 \pm 21.5$

0.483

$26.7 \pm 26.5$

0.288

$29.8 \pm 23.0$

0.464

$22.9 \pm 26.2$

0.460

$29.4 \pm 25.0$

0.215

$34.6 \pm 29.7$

0.110

$23.9 \pm 26.6$

0.706

$23.2 \pm 22.1$

0.052a

$32.0 \pm 25.1$

0.547

$36.1 \pm 26.9$

0.279

$27.3 \pm 23.5$

0.249

$34.2 \pm 25.0$

0.169

$35.7 \pm 24.9$

0.510

$34.3 \pm 28.4$

0.189

$37.2 \pm 32.4$

0.343

$36.9 \pm 29.2$

$0.038^{b}$

$25.8 \pm 22.3$

0.168

$37.4 \pm 26.0$

0.092

$40.8 \pm 30.6$

0.166

$32.8 \pm 25.0$

0.423

$36.3 \pm 24.8$

0.321

a: post-hoc analyses indicate a trend for a significant difference only between Low and Good responders with a Bonferroni corrected $p$-value $=0.073$; $b$ : post-hoc analyses indicate a significant difference only between Low and Good responders with a Bonferroni corrected $\mathrm{p}$-value $=0.033$; c: b: all p-value for the post-hoc analyses were $>0.1$ after Bonferroni correction. 
Table 5: Relationship between implicit wanting for foods regarding percentage of Total Weight Loss tercile groups.

\section{Low responders}

High carb - Solid

High carb - Fluid

Low carb - Solid

Low carb - Fluid

Dairy - Color

Dairy - No color

Nondairy - Color

Nondairy - No color

High fat - Savory

High fat - Sweet

Low fat - Savory

Low fat - Sweet

Fiber - Sauce

Fiber - No sauce

No fiber - Sauce

No fiber - No sauce

Meat - High fat

Meat - Low fat

No meat - High fat

No meat - Low fat

High protein - Variation

High protein - No variation

Low protein - Variation

Low protein - No variation

$$
\begin{aligned}
& <33 \% \\
& n=18
\end{aligned}
$$

mean $S D$

Middle responders

$$
33-66 \%
$$$$
n=19
$$

$0.94 \pm 27.62$

$-7.82 \pm 21.69$

$15.84 \pm 27.92$

$-8.96 \pm 33.35$

$-2.95 \pm 17.49$

$23.14 \pm 29.23$

$-24.27 \pm 29.86$

$4.08 \pm 24.58$

$2.86 \pm 28.82$

$-4.41 \pm 30.22$

$-11.87 \pm 38.09$

$15.41 \pm 23.65$

$0.79 \pm 20.09$

$-14.02 \pm 26.57$

$-6.13 \pm 22.25$

$18.33 \pm 22.80$

$11.10 \pm 22.44$

$11.94 \pm 18.73$

$-11.69 \pm 20.54$

$-5.49 \pm 32.93$

$-5.30 \pm 18.12$

$5.25 \pm 18.82$

$-1.93 \pm 17.00$

$1.98 \pm 16.08$

\section{Good responders}

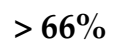

ANOVA

$p$ value

$-5.98 \pm 22.97$

0.744

$-6.92 \pm 18.35$

0.828

$34.78 \pm 15.74$

$\mathbf{0 . 0 1 1}^{\mathrm{a}}$

$-21.88 \pm 30.60$

0.222

$-2.74 \pm 26.68$

0.804

$22.36 \pm 18.97$

0.846

$-27.71 \pm 15.79$

0.163

$8.10 \pm 29.33$

0.345

$19.26 \pm 25.88$

$0.071^{b}$

$-8.23 \pm 20.57$

0.619

$-16.33 \pm 28.15$

0.819

$5.30 \pm 20.49$

0.321

$-2.34 \pm 24.25$

0.705

$-16.39 \pm 23.48$

0.317

$3.86 \pm 15.33$

0.164

$14.88 \pm 20.46$

0.359

$10.32 \pm 15.20$

0.287

$7.98 \pm 18.81$

0.605

$6.46 \pm 13.73$

$0.046^{c}$

$-24.75 \pm 18.28$

$0.095^{\mathrm{d}}$

$1.89 \pm 18.25$

0.635

$21.69 \pm 20.87$

$0.038^{\mathrm{e}}$

$-14.85 \pm 14.16$

$0.064^{\mathrm{f}}$

$-8.73 \pm 17.68$

0.206

a: post-hoc analyses indicate a significant difference only between Low and Good responders with a Bonferroni corrected $p$-value $=0.011 ; b$ : all $p$-value for the post-hoc analyses were $>0.1$ after Bonferroni correction; c: post-hoc analyses indicate a significant difference only between Low and Good responders with a Bonferroni corrected p-value $=0.043$; $\mathrm{d}$ : all p-value for the post-hoc analyses were $>0.1$ after Bonferroni correction; e: post-hoc analyses indicate a trend for a significant difference only between Low and good Good responders with a Bonferroni corrected p-value = 0.061; f: post-hoc analyses indicate a trend for a significant difference only between Low and Good responders with a Bonferroni corrected $\mathrm{p}$-value $=0.076$. 


\section{Discussion}

The present exploratory study aimed to investigate thoroughly liking and wanting for food items (i.e., food reward components) in participants with a bariatric surgery using a computerized behavioral task. The originality of this study relies on the use of a wide range of food items varying in nutrition composition, appearance, taste, and texture and on its comparative design considering the types of surgery (SG and RYGB) and the weight loss outcome.

The present work is among the few studies using a distinct comparative design towards the two most common types of bariatric surgery. Regarding socio-demographic data, participants with SG or RYGB did not differ significantly except for age (SG $=38.3$ years and RYGB $=50.5$ years, $\mathrm{p}<0.001$ ). Age and gender influence on taste has been documented and might have influenced our results [34]. Although statistically significant, this difference appears to be of small clinical relevance given that all patients belong to the same middle-age category and may not influence food preferences. Our findings suggest that there is no major difference between the two types of surgery regarding liking and wanting for all foods categories assessed. This is in accordance with studies, showing a similar decrease in preference for fat [35] and liking for high calorie dense foods [36], as well as an increase in diet quality [37] and dietary intake [38] after both SG and RYGB. However, another study showed a decrease in the hedonic rating for high fat after SG and RYGB, but the liking of high sugar remained unchanged after RYGB [35]. RYGB induces more dumping syndrome than SG [39], and thus the digestive discomfort caused by the consumption of foods rich in carbohydrates may have resulted in stronger conditioned taste avoidance in RYGB than in SG. Although this is a frequent issue in clinical practice, our results do not indicate any relevant changes in relation to carbohydrates liking or wanting, in any form (i.e., solid or liquid).

Considering weight loss outcomes (assessed by \%TWL), comparisons of explicit liking and, explicit and implicit wanting scores between low and good weight loss responders suggest differences in reward depending on food protein content. In our study, participants with a better weight loss trajectory tend to prefer foods with higher protein content. Interestingly, these results could be explained within the framework of the protein leverage hypothesis. This hypothesis was primarily developed to explain overeating in the context of obesity [40-42]. This theory assumes that protein intake is prioritized over carbohydrates and fat intake. Accordingly, participants eat until their protein needs are met, regardless of the energy content of the food. Thus, participants with higher weight loss may have a stronger desire (and consumption) for protein and therefore, consume less palatable products (with high energy density but low protein content). Further confirmation of such findings in a prospective study would be of importance, especially to determine whether a higher pre-surgery protein wanting causes a greater weight loss or whether such modifications appear after bariatric surgery.

In contrast to our expectations, patients with lower weight loss did not show higher liking or wanting for products rich in fat or carbohydrates, except for 'Low carb - Solid' implicit wanting. A systematic review showed that food reward decreased during dietary, exercise, pharmacological, cognitive and behavioral/multidisciplinary weight loss interventions [43]. This suggests that more than the bariatric surgery itself, the weight loss and therefore the energy imbalance might have an impact on the motivation to eat and food choices. Most patients experience a "honeymoon" in the first post-operative year (12-18 months), where they have a reduced appetite, limiting portion sizes, and disinterest in foods [44], which is concomitant with the great and rapid weight loss phase. Our results might be explained considering the time points of assessment reflecting the time since surgery. Indeed, in a previous study, we observed that participants had a higher appreciation of highly palatable foods after 2 years of follow-up than those having been operated 
more recently [13]. Other studies also suggest a return of dietary preferences to prior patterns with increasing time since surgery $[37,45,46]$. Using the Leeds Food Preference Questionnaire, Oustric et al. also observed such a backward step after weight loss programs [47]. Prospective and long-term assessments (after 2 years of follow-up) of food rewards are necessary to better understand if those modifications are sustained and/or if they are related to the weight regain that occurs in $20 \%$ of patients within 1 to 3 years after surgery [48].

Some limitations must be considered, especially the cross-sectional design of the study and the small sample size. The transversal design does not allow us to test the causal effect of bariatric surgery on the differences of food reward found between lower weight loss responders and good or normal weight loss responders. The relatively small sample size and the sampling method (routine day care) is also an important limitation, especially considering the important number of statistical analyses conducted. Larger sample sizes are needed to achieve the required power to apply a proper correction for multiple testing. Another limitation is the lack of controlled laboratory conditions when measuring food preferences as the visits occurred in the hospitalization unit and the time of arrival of the patients were based on their pre-registered clinical consultancy, which prevented from rigorously standardizing fasting status, appetite levels and time since the last meal in patients. However, when comparing the type of surgery or weight loss trajectory status, the time from last meal or appetite sensations did not differ between groups. Our results showed a significant difference in age between patients with SG and RYGB ( 38 vs. 51 years old respectively). Finally, food preferences could have been influenced by other parameters, such as the socio-cultural background and ethnicity, which could be considered in future research given their influence on food choices [49,50]. Despite these limitations, our study, by addressing a wider range of food items, highlights the need to consider adding protein items in future research on food rewards, especially in bariatric surgery field.

\section{Conclusions}

To conclude, liking and wanting for a comprehensive range of food items were not different in participants with either SG or RYGB. Wanting for protein items might be related to the weight loss response after bariatric surgery, while no such association was found for usual palatable foods. Adding protein items to usual palatable items could be clinically relevant to identify postoperative alterations in food reward and guide caregivers to give personalized advice to patients in the context of precision bariatric medicine.

Supplementary Materials: The following are available online at www.mdpi.com/xxx/s1, Table S1 : Relationship between liking and wanting for foods and bariatric surgery type; Table S2 : Table S2 : Socioeconomic and behavioral characteristics by Total Weight Loss tercile.

Author Contributions: Conceptualization: S.I., E.D.; methodology, E.G., S.I. and A.D.; software, E.G. and P.O.; validation, S.I., E.D., J-A.N. and A.D.; formal analysis, E.G., S.I.; investigation, E.G., S.I.; data curation, E.G.; writing - original draft preparation, S.I., E.G.; writing - review and editing, E.G. S.I., E.D., J-A.N., A.D., P.O. and M.R.; supervision, S.I., E.D., J-A.N. and A.D.; funding acquisition, S.I., E.D. All authors have read and agreed to the published version of the manuscript.

Funding: This research received no external funding.

Institutional Review Board Statement: The study was conducted according to the guidelines of the Declaration of Helsinki and approved by a national ethic Committee (2017-A02953-50, on January 16th, 2018) and was registered in Clinical Trials.gov (NCT03486210).

Informed Consent Statement: Written informed consent was obtained from all subjects involved in the study.

Data Availability Statement: The data presented in this study are available on reasonable request from the corresponding author. The data are not publicly available.

Acknowledgments: The authors gratefully thank Graham Finlayson, Monique Sothier, Laurie Vandenberghe, Julien Ferretti and Estelle Petit for their help in the adaptation of the LFPQ for French culture. We thank Brice Mastrovito, Julie Renault and Sonia Balleydier for helping with recruitment and data collection. The authors thanks all the patients participating in this study. 
Conflicts of Interest: Mrs. Guyot reports grants from Agence Nationale Recherche et Technologie (ANRT), from Fondation APICIL and, from Institut Benjamin Delessert. The other authors declare that there is no conflict of interest regarding the publication of this article. The content of the work is the sole responsibility of the authors and do not necessarily represent the views of the funders. 


\section{References}

1. Sjöström, L.; Peltonen, M.; Jacobson, P.; Sjöström, C.D.; Karason, K.; Wedel, H.; Ahlin, S.; Anveden, Å.; Bengtsson, C.; Bergmark, G.; et al. Bariatric Surgery and Long-Term Cardiovascular Events. JAMA - J. Am. Med. Assoc. 2012, 307, 56-65, doi:10.1001/jama.2011.1914.

2. Sjöström, L.; Narbro, K.; Sjöström, C.D.; Karason, K.; Larsson, B.; Wedel, H.; Lystig, T.; Sullivan, M.; Bouchard, C.; Carlsson, B.; et al. Effects of Bariatric Surgery on Mortality in Swedish Obese Subjects. N. Engl. J. Med. 2007, 357, 741-752, doi:10.1056/NEJMoa066254.

3. Sjöström, L. Review of the Key Results from the Swedish Obese Subjects (SOS) Trial - a Prospective Controlled Intervention Study of Bariatric Surgery. J. Intern. Med. 2013, 273, 219-234.

4. Angrisani, L.; Santonicola, A.; Iovino, P.; Vitiello, A.; Zundel, N.; Buchwald, H.; Scopinaro, N. Bariatric Surgery and Endoluminal Procedures: IFSO Worldwide Survey 2014. Obes. Surg. 2017, 27, 1-11, doi:10.1007/s11695-017-2666-x.

5. Roth, A.E.; Thornley, C.J.; Blackstone, R.P. Outcomes in Bariatric and Metabolic Surgery: An Updated 5-Year Review. Curr. Obes. Rep. 2020, 9, 380-389, doi:10.1007/S13679-020-00389-8.

6. Coluzzi, I.; Raparelli, L.; Guarnacci, L.; Paone, E.; Del Genio, G.; le Roux, C.W.; Silecchia, G. Food Intake and Changes in Eating Behavior After Laparoscopic Sleeve Gastrectomy. Obes. Surg. 2016, 26, 2059-2067, doi:10.1007/s11695-015-2043-6.

7. Laurenius, A.; Larsson, I.; Melanson, K.J.; Lindroos, A.K.; Lönroth, H.; Bosaeus, I.; Olbers, T. Decreased Energy Density and Changes in Food Selection Following Roux-En-Y Gastric Bypass. Eur. J. Clin. Nutr. 2013, 67, 168-173, doi:10.1038/ejcn.2012.208.

8. Thirlby, R.C.; Bahiraei, F.; Randall, J.; Drewnoski, A. Effect of Roux-En-Y Gastric Bypass on Satiety and Food Likes: The Role of Genetics. J. Gastrointest. Surg. Off. J. Soc. Surg. Aliment. Tract 2006, 10, 270-277, doi:10.1016/j.gassur.2005.06.012.

9. Guyot, E.; Dougkas, A.; Bagot, S.; Nazare, J.A.; Disse, E.; Iceta, S. A Systematic Review of Food Preference Modifications after Bariatric Surgery. Obes. Rev., doi:10.1111/obr.13315.

10. Peng, M.; Coutts, D.; Wang, T.; Cakmak, Y.O. Systematic Review of Olfactory Shifts Related to Obesity. Obes. Rev. Off. J. Int. Assoc. Study Obes. 2019, 20, 325-338, doi:10.1111/obr.12800.

11. Ahmed, K.; Penney, N.; Darzi, A.; Purkayastha, S. Taste Changes after Bariatric Surgery: A Systematic Review. Obes. Surg. 2018, 28, 3321-3332, doi:10.1007/s11695-018-3420-8.

12. Nielsen, M.S.; Andersen, I.N.S.K.K.; Lange, B.; Ritz, C.; le Roux, C.W.; Schmidt, J.B.; Sjodin, A.; Bredie, W.L.P.P.; Sjödin, A.; Bredie, W.L.P.P.; et al. Bariatric Surgery Leads to Short-Term Effects on Sweet Taste Sensitivity and Hedonic Evaluation of Fatty Food Stimuli. Obesity 2019, doi:10.1002/oby.22589.

13. Guyot, E.; Dougkas, A.; Robert, M.; Nazare, J.-A.; Iceta, S.; Disse, E. Food Preferences and Their Perceived Changes Before and After Bariatric Surgery: A Cross-Sectional Study. Obes. Surg. 2021, doi:10.1007/s11695-021-05342-9.

14. Berridge, K.C. Food Reward: Brain Substrates of Wanting and Liking. Neurosci. Biobehav. Rev. 1996, 20, 1-25, doi:10.1016/01497634(95)00033-B.

15. Tibboel, H.; De Houwer, J.; Van Bockstaele, B. Implicit Measures of "Wanting" and "Liking" in Humans. Neurosci. Biobehav. Rev. 2015, 57, 350-364, doi:10.1016/j.neubiorev.2015.09.015.

16. Berridge, K.C.; Robinson, T.E. Parsing Reward. Trends Neurosci. 2003, 26, 507-513, doi:10.1016/S0166-2236(03)00233-9.

17. Finlayson, G.; King, N.; Blundell, J.E. Is It Possible to Dissociate "liking" and "Wanting" for Foods in Humans? A Novel Experimental Procedure. Physiol. Behav. 2007, 90, 36-42, doi:10.1016/j.physbeh.2006.08.020.

18. Oustric, P.; Thivel, D.; Dalton, M.; Beaulieu, K.; Gibbons, C.; Hopkins, M.; Blundell, J.; Finlayson, G. Measuring Food Preference and Reward: Application and Cross-Cultural Adaptation of the Leeds Food Preference Questionnaire in Human Experimental Research. Food Qual. Prefer. 2020, 103824, doi:10.1016/j.foodqual.2019.103824.

19. Goldstone, A.P.; Miras, A.D.; Scholtz, S.; Jackson, S.; Neff, K.J.; Pénicaud, L.; Geoghegan, J.; Chhina, N.; Durighel, G.; Bell, J.D.; et al. Link between Increased Satiety Gut Hormones and Reduced Food Reward after Gastric Bypass Surgery for Obesity. J. Clin. Endocrinol. Metab. 2016, 101, 599-609, doi:10.1210/jc.2015-2665. 
20. Scholtz, S.; Miras, A.D.; Chhina, N.; Prechtl, C.G.; Sleeth, M.L.; Daud, N.M.; Ismail, N.A.; Durighel, G.; Ahmed, A.R.; Olbers, T.; et al. Obese Patients after Gastric Bypass Surgery Have Lower Brain-Hedonic Responses to Food than after Gastric Banding. Gut 2014, 63, 891-902, doi:10.1136/gutjnl-2013-305008.

21. Ochner, C.N.; Kwok, Y.; Conceicao, E.; Pantazatos, S.P.; Puma, L.M.; Carnell, S.; Teixeira, J.; Hirsch, J.; Geliebter, A. Selective Reduction in Neural Responses to High Calorie Foods Following Gastric Bypass Surgery. Ann. Surg. 2011, 253, 502-507, doi:10.1097/SLA.0b013e318203a289.

22. Ochner, C.N.; Stice, E.; Hutchins, E.; Afifi, L.; Geliebter, A.; Hirsch, J.; Teixeira, J. Relation between Changes in Neural Responsivity and Reductions in Desire to Eat High-Calorie Foods Following Gastric Bypass Surgery. Neuroscience 2012, 209, 128-135, doi:10.1016/j.neuroscience.2012.02.030.

23. Frank, S.; Veit, R.; Sauer, H.; Enck, P.; Friederich, H.C.; Unholzer, T.; Bauer, U.M.; Linder, K.; Heni, M.; Fritsche, A.; et al. Dopamine Depletion Reduces Food-Related Reward Activity Independent of BMI. Neuropsychopharmacology 2016, 41, 15511559, doi:10.1038/npp.2015.313.

24. Frank, S.; Heinze, J.M.; Fritsche, A.; Linder, K.; von Feilitzsch, M.; Konigsrainer, A.; Haring, H.-U.; Veit, R.; Preissl, H.; Königsrainer, A.; et al. Neuronal Food Reward Activity in Patients With Type 2 Diabetes With Improved Glycemic Control After Bariatric Surgery. Diabetes Care 2016, 39, 1311-1317, doi:10.2337/dc16-0094.

25. Coveleskie, K.; Gupta, A.; Kilpatrick, L.A.; Mayer, E.D.; Ashe-Mcnalley, C.; Stains, J.; Labus, J.S.; Mayer, E.A. Altered Functional Connectivity within the Central Reward Network in Overweight and Obese Women. Nutr. Diabetes 2015, 5, doi:10.1038/nutd.2014.45.

26. Stice, E.; Spoor, S.; Bohon, C.; Veldhuizen, M.G.; Small, D.M. Relation of Reward From Food Intake and Anticipated Food Intake to Obesity: A Functional Magnetic Resonance Imaging Study. J. Abnorm. Psychol. 2008, 117, 924-935, doi:10.1037/a0013600.

27. Sanjur, D.; Randall, E.; Sanjur, D. Food Preferences-Their Conceptualization and Relationship to Consumption. Ecol. Food Nutr. 1981, 11, 151-161, doi:10.1080/03670244.1981.9990671.

28. Stano, S.; Alam, F.; Wu, L.; Dutia, R.; Ng, S.N.; Sala, M.; McGinty, J.; Laferrère, B. Effect of Meal Size and Texture on Gastric Pouch Emptying and Glucagon-like Peptide 1 after Gastric Bypass Surgery. Surg. Obes. Relat. Dis. 2017, 13, 1975-1983, doi:10.1016/j.soard.2017.09.004.

29. Smith, K.R.; Papantoni, A.; Veldhuizen, M.G.; Kamath, V.; Harris, C.; Moran, T.H.; Carnell, S.; Steele, K.E. Taste-Related Reward Is Associated with Weight Loss Following Bariatric Surgery. J. Clin. Invest. 2020, 130, 4370-4381, doi:10.1172/JCI137772.

30. van der Meij, B.S.; Wijnhoven, H.A.H.; Finlayson, G.S.; Oosten, B.S.H.; Visser, M. Specific Food Preferences of Older Adults with a Poor Appetite. A Forced-Choice Test Conducted in Various Care Settings. Appetite 2015, 90, 168-175, doi:10.1016/j.appet.2015.03.011.

31. Park, J.Y.; Kim, Y.J. Validation of the Alterable Weight Loss Metric in Morbidly Obese Patients Undergoing Gastric Bypass in Korea. Obes. Surg. 2018, 28, 1704-1710, doi:10.1007/s11695-017-3084-9.

32. Tu, Y.; Pan, Y.; Han, J.; Pan, J.; Zhang, P.; Jia, W.; Bao, Y.; Yu, H. A Total Weight Loss of 25\% Shows Better Predictivity in Evaluating the Efficiency of Bariatric Surgery. Int. J. Obes. 2005 2021, 45, 396-403, doi:10.1038/s41366-020-00690-5.

33. van de Laar, A.W.; de Brauw, L.M.; Meesters, E.W. Relationships between Type 2 Diabetes Remission after Gastric Bypass and Different Weight Loss Metrics: Arguments against Excess Weight Loss in Metabolic Surgery. Surg. Obes. Relat. Dis. 2016, 12, 274-282, doi:10.1016/j.soard.2015.07.005.

34. Barragán, R.; Coltell, O.; Portolés, O.; Asensio, E.M.; Sorlí, J.V.; Ortega-Azorín, C.; González, J.I.; Sáiz, C.; Fernández-Carrión, R.; Ordovas, J.M.; et al. Bitter, Sweet, Salty, Sour and Umami Taste Perception Decreases with Age: Sex-Specific Analysis, Modulation by Genetic Variants and Taste-Preference Associations in 18 to 80 Year-Old Subjects. Nutrients 2018, 10, E1539, doi:10.3390/nu10101539. 
35. Primeaux, S.D.; Tzeng, T.H.; Allerton, T.D.; Chiang, M.C.; Cosentino, G.; Dubin, R.L.; Varughese, A.; Moore, R.; Geiselman, P.J.; Greenway, F.L.; et al. Differences in Short-Term Food Preferences Following Vertical Sleeve Gastrectomy and Roux-En-Y Gastric Bypass Surgery. Obes. Res. Clin. Pract. 2015, 9, 628-632.

36. Faulconbridge, L.F.; Ruparel, K.; Loughead, J.; Allison, K.C.; Hesson, L.A.; Fabricatore, A.N.; Rochette, A.; Ritter, S.; Hopson, R.D.; Sarwer, D.B.; et al. Changes in Neural Responsivity to Highly Palatable Foods Following Roux-En-Y Gastric Bypass, Sleeve Gastrectomy, or Weight Stability: An FMRI Study. Obesity 2016, doi:10.1002/oby.21464.

37. Hubert, P.A.; Papasavas, P.; Stone, A.; Swede, H.; Huedo-Medina, T.B.; Tishler, D.; Duffy, V.B. Associations between Weight Loss, Food Likes, Dietary Behaviors, and Chemosensory Function in Bariatric Surgery: A Case-Control Analysis in Women. Nutrients 2019, 11, 1-23, doi:10.3390/nu11040804.

38. El Labban, S.; Safadi, B.; Olabi, A. The Effect of Roux-En-Y Gastric Bypass and Sleeve Gastrectomy Surgery on Dietary Intake, Food Preferences, and Gastrointestinal Symptoms in Post-Surgical Morbidly Obese Lebanese Subjects: A Cross-Sectional Pilot Study. Obes. Surg. 2015, 25, 2393-2399, doi:10.1007/s11695-015-1713-8.

39. Ramadan, M.; Loureiro, M.; Laughlan, K.; Caiazzo, R.; Iannelli, A.; Brunaud, L.; Czernichow, S.; Nedelcu, M.; Nocca, D. Risk of Dumping Syndrome after Sleeve Gastrectomy and Roux-En-Y Gastric Bypass: Early Results of a Multicentre Prospective Study. Gastroenterol. Res. Pract. 2016, 2016, doi:10.1155/2016/2570237.

40. Simpson, S.J.; Raubenheimer, D. Obesity: The Protein Leverage Hypothesis. Obes. Rev. 2005, 6, $133-142$.

41. Raubenheimer, D.; Simpson, S.J. Protein Leverage: Theoretical Foundations and Ten Points of Clarification. Obesity 2019, 27, 1225-1238.

42. Champeil-Potokar, G.; Crossouard, L.; Jérôme, N.; Ouali, C.; Darcel, N.; Davidenko, O.; Rampin, O.; Bombail, V.; Denis, I. Diet Protein Content and Individual Phenotype Affect Food Intake and Protein Appetence in Rats. J. Nutr. 2021, doi:10.1093/jn/nxaa455.

43. Oustric, P.; Gibbons, C.; Beaulieu, K.; Blundell, J.; Finlayson, G. Changes in Food Reward during Weight Management Interventions - a Systematic Review. Obes. Rev. 2018, 19, 1642-1658.

44. Lynch, A. "When the Honeymoon Is over, the Real Work Begins:" Gastric Bypass Patients' Weight Loss Trajectories and Dietary Change Experiences. Soc. Sci. Med. 2016, 151, 241-249, doi:10.1016/j.socscimed.2015.12.024.

45. Kittrell, H.; Graber, W.; Mariani, E.; Czaja, K.; Hajnal, A.; Lorenzo, P.M.D. Taste and Odor Preferences Following Roux-En-Y Surgery in Humans. PLOS ONE 2018, 13, e0199508, doi:10.1371/journal.pone.0199508.

46. Brolin, R.E.; Robertson, L.B.; Kenler, H.A.; Cody, R.P. Weight Loss and Dietary Intake After Vertical Banded Gastroplasty and Roux-En-Y Gastric Bypass. Ann. Surg. 1994, 220, 782-790.

47. Oustric, P.; Beaulieu, K.; Casanova, N.; O’Connor, D.; Gibbons, C.; Hopkins, M.; Blundell, J.; Finlayson, G. Food Liking but Not Wanting Decreases after Controlled Intermittent or Continuous Energy Restriction to $\geq 5 \%$ Weight Loss in Women with Overweight/Obesity. Nutrients 2021, 13, doi:10.3390/nu13010182.

48. Sjöström, L.; Lindroos, A.K.; Peltonen, M.; Torgerson, J.; Bouchard, C.; Carlsson, B.; Dahlgren, S.; Larsson, B.; Narbro, K.; Sjöström, C.D.; et al. Lifestyle, Diabetes, and Cardiovascular Risk Factors 10 Years after Bariatric Surgery. N. Engl. J. Med. 2004, 351, 2683-2693, doi:10.1056/NEJMoa035622.

49. Rozin, P. The Socio-Cultural Context of Eating and Food Choice. In Food choice, acceptance and consumption; Springer, 1996; pp. 83-104.

50. Enriquez, J.P.; Archila-Godinez, J.C. Social and Cultural Influences on Food Choices: A Review. Crit. Rev. Food Sci. Nutr. 2021, $1-7$. 